Artículo

\title{
Evaluación financiera de producción de agave y mezcal: caso de estudio Caltepec, Puebla
}

\author{
Mariana Fonseca Varela \\ Luis Eduardo Chalita Tovar ${ }^{\S}$ \\ Estadística e Informática y Economía-Postgrado en Economía-Colegio de Postgraduados-Campus \\ Montecillo. Carretera México-Texcoco km 36.5, Montecillo, Texcoco, Estado de México. CP. 56230. Tel. \\ 595 9520284. (economia@colpos.mx). \\ ${ }^{\S}$ Autor de correspondencia: chalita@colpos.mx.
}

\section{Resumen}

En los últimos años la producción de destilados de agave en México, dentro de ellos el mezcal, se ha vuelto cada vez más importante. En el periodo 2013-2019, su valor ha aumentado a una tasa promedio anual de $19.76 \%$. Tal comportamiento ha sido impulsado por los precios del producto y la creciente demanda nacional e internacional, respecto a este último concepto de 2017 a 2018 las exportaciones de mezcal crecieron a una tasa de $34.4 \%$. Lo anterior, ha llevado a que cada vez más unidades productivas se vinculen a la cadena de valor del agave, como ha sido el caso del municipio de Caltepec, Puebla, cuya población se encuentra en pobreza y vive de la agricultura. En este sentido, es necesario analizar las oportunidades que pudiera generar la producción de agave y mezcal, para proveer oportunidades productivas que busquen mejorar la economía municipal. Con base en lo anterior, se elaboró una evaluación financiera a partir del año 2020 con un periodo de planeación de ocho y 15 años a partir de la puesta en marcha del proyecto. Los hallazgos de la investigación sugieren que ambos tipos de oportunidades de negocio son factibles financieramente, al obtener un valor actual neto de \$34 074.09 y \$1 911 792.96, respectivamente. Por lo que pueden servir como alternativas potenciales a mejorar el bienestar de distintas unidades productivas de la región, con ello coadyubando a mitigar la pobreza local.

Palabras clave: Agave potatorum, análisis financiero, agronegocio, rentabilidad.

Recibido: enero de 2021

Aceptado: marzo de 2021 


\section{Introducción}

El agave es la materia prima para la elaboración de distintas bebidas destiladas, dentro de ellas el mezcal, cuya denominación de origen es mexicana y se considera una bebida espirituosa de alta graduación (Cárcamo y Noriega, 2009). Las exportaciones de este producto alcanzaron en 2016 los 2013 miles de litros, que significaron un ingreso de 26 millones de dólares al país (SAGARPA, 2017); además, las ventas al resto del mundo aumentaron en 2018 un 34.4\% respecto al año inmediato anterior (INEGI, 2020).

De acuerdo con Pérez et al. (2016), en 2014, México contaba con cerca de 330000 ha de agave en explotación, propiedad de 9000 productores, que generaban 29000 empleos directos e indirectos. Para el caso del mezcal, en 2006 existían 625 fábricas, 80 plantas envasadoras y 130 marcas de mezcal. Para 2014 el número de marcas registradas ascendió a 362 y la producción de mezcal aumentó 48\% comparado con el dato de 2011.

De acuerdo con cifras del Sistema de Información Agroalimentaria de Consulta (SIACON, 2020) la producción y superficie sembrada de agave mezcalero ha crecido en los últimos diez años de 2008 a 2018 la producción pasó de 301790 a 303382 t, mientras que la superficie sembrada lo hizo de 4700 a 5360 ha. Aun cuando existen avances en el cultivo del agave y mejoría en las condiciones de ofertantes y demandantes, la agroindustria del mezcal enfrenta retos y limitaciones en los diferentes eslabones de la cadena (AGARED, 2017). Empero, la dinámica observada por el lado de la oferta indica que cada vez más unidades productivas se han vinculado a la cadena de valor del agave y sus destilados.

Este ha sido el caso de Puebla, México, se considera que el estado cuenta con un potencial superior a 250000 ha para el cultivo de agave en el centro-sur de su territorio (Jiménez-Merino, 2017; Ulloa y Medrano, 2019).

La superficie sembrada de agave en Puebla aumentó 67.33\% entre 2008 y 2018. Sin embargo, dicha producción de agave mezcalero se concentra en pocos municipios, dentro de ellos San Diego La Mesa Tochimiltzingo, Huehuetlán El Grande y Tecali de Herrera con 50, 40 y 30 ha, respectivamente. Lo anterior a pesar de que en otros municipios del estado existen condiciones idóneas para su producción.

Como parte de los agaves mezcaleros aptos o endémicos de Puebla se encuentra el Agave potatorum, que se distribuye en tierras semiáridas de las partes altas del estado, en altitudes que van de 1240 a 2300 msnm, en laderas con vegetación herbácea densa y remanentes de vegetación de pino y encino. En las laderas que se extienden a lo largo de la carretera Puebla-Oaxaca, cerca de Yanhuitlán, usualmente son visibles los ejemplares de Agave potatorum de rosetas pequeñas e inflorescencias racimosas (García, 2010).

Dentro de las regiones poblanas con idoneidad para la producción del Agave potatorum se encuentra el municipio de Caltepec, zona considerada marginada, donde más de $70 \%$ de su población vive en pobreza y tiene como principal medio de generación de ingresos a la agricultura (CONEVAL, 2010). En el Cuadro 1 se disponen algunos indicadores relevantes. 
Cuadro 1. Índice de desarrollo humano de Caltepec, Puebla.

\begin{tabular}{lcc}
\hline Índice & Personas & $(\%)$ \\
\hline Población total & 3704 & 100 \\
Población situación pobreza & 2792 & 75.37 \\
Pobreza extrema & 990 & 26.73 \\
Pobreza extrema sin alimentación & 434 & 11.37 \\
Pobreza moderada & 1802 & 48.64 \\
Vulnerables por carencia social & 853 & 23.04 \\
Vulnerables por ingreso & 33 & 0.9 \\
No pobres y no vulnerables & 26 & 0.7 \\
\hline
\end{tabular}

CONEVAL (2010).

Sobre este marco surge la necesidad e interés de analizar las oportunidades y beneficios que pudiera traer consigo la puesta en marcha de fincas productoras de agave y mezcal en la región, cuya práctica se ha perfilado como una fuente alternativa de ingresos en lugares marginados (Rincón $e t$ $a l .$, 2015). Con ello, se buscó identificar oportunidades de negocio que busquen mejorar la economía municipal y abrir un menú de opciones para que los campesinos decidan, en consideración de los apoyos federales y estatales, diversificar sus fuentes de ingreso, que actualmente se encuentran limitados a la producción de gramíneas.

Por ende, el objetivo de la investigación fue calcular la rentabilidad de la producción de agave y mezcal en Caltepec, Puebla. Para ello se consideró un periodo de planeación de ocho y 15 años, respectivamente y se contrastaron los retornos de los proyectos con los obtenidos actualmente de la producción de maíz, lo que permitió concluir sobre su factibilidad.

\section{Materiales y métodos}

El estudio se realizó para el municipio de Caltepec, Puebla (Figura 1), el cual se localiza entre los paralelos $18^{\circ} 02^{\prime}$ y $18^{\circ} 16^{\prime}$ de latitud norte; los meridianos $97^{\circ} 21^{\prime}$ y $97^{\circ} 39^{\prime}$ de longitud oeste. Colinda al noreste con San José Miahuatlán y de oeste a norte con Zapotitlán, al sureste con los municipios de Tepelmeme Villa de Morelos, al oeste con Santiago de Chazumba, al sur Santa Catarina Zapoquila y Concepción Buenavista, al suroeste San Pedro y San Pablo Tequixtepec (INFADED, 2019).

El clima predominantemente semicálido de la región es apto para el Agave potatorum, que crece de forma silvestre en las partes altas del municipio. De acuerdo con Cruz (2019) las prácticas técnicas y culturales habituales para la producción de agave en Caltepec son: 1) barbecho; 2) rastra; 3) cruza; 4) levantamiento de camas; 5) preparación de la semilla; 6) siembra; 7) transplante; 8) deshierbe; 9) aporque; 10) fertilización; 11) control fitosanitario; y 12) cosecha (hecha a menudo a manos del comprador). La información de la estructura de costos antes señalada fue obtenida en campo, en el Cuadro 2 se dispone la información obtenida por hectárea. 


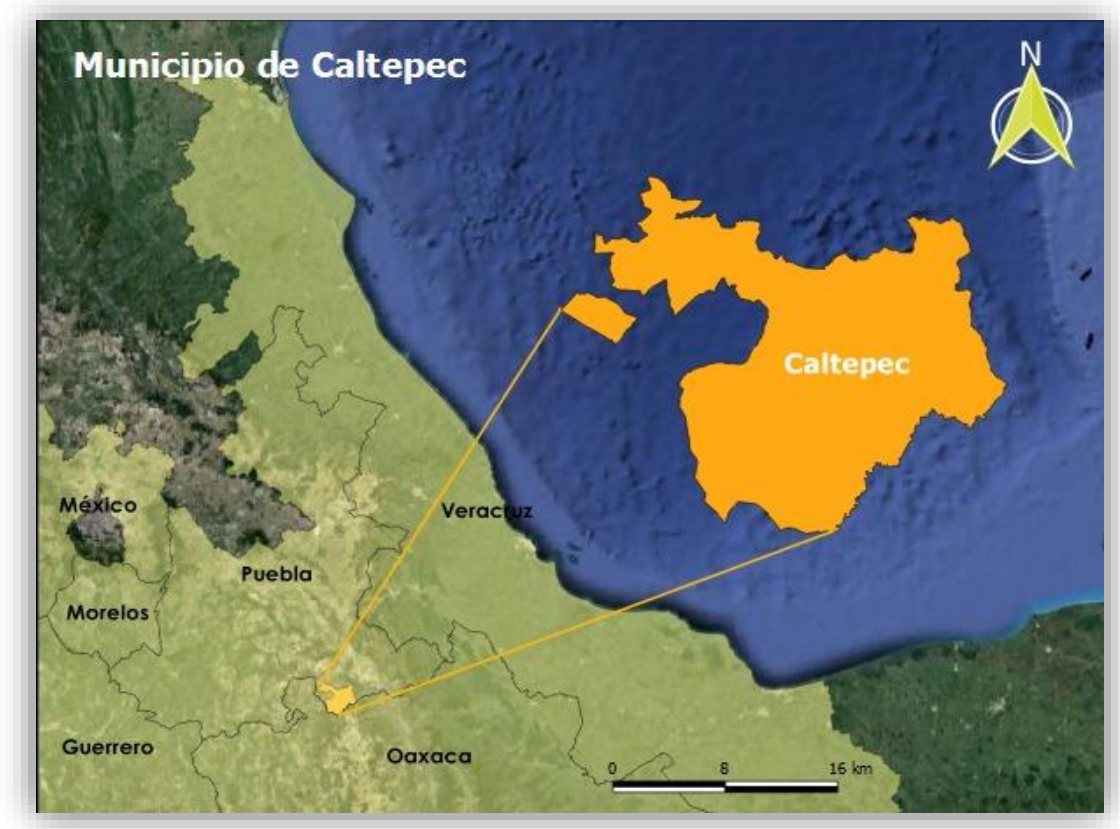

Figura 1. Área de estudio, Caltepec, Puebla, México. Elaboración con datos de google earth.

Cuadro 2. Estructura de costos de la producción de agave (miles de pesos).

\begin{tabular}{|c|c|c|c|c|c|c|c|c|c|}
\hline \multirow{2}{*}{ Actividades realizadas en el ciclo } & \multicolumn{8}{|c|}{ Año } & \multirow{2}{*}{$\begin{array}{c}\text { Costo total } \\
(\$)\end{array}$} \\
\hline & 1 & 2 & 3 & 4 & 5 & 6 & 7 & 8 & \\
\hline Renta de la tierra por hectárea & & & & & & & & & 0.30 \\
\hline Asesoría técnica & $*$ & $*$ & $*$ & $*$ & $*$ & $*$ & $*$ & $*$ & 3.00 \\
\hline Trazo del marco con curva de nivel & $*$ & & & & & & & & 0.50 \\
\hline Cajeteo & $*$ & $*$ & $*$ & * & * & * & $*$ & & 16.80 \\
\hline Compra de plántula & $*$ & & & & & & & & 4.80 \\
\hline Flete del vivero al lugar de siembra & $*$ & & & & & & & & 0.30 \\
\hline Transplante & $*$ & & & & & & & & 0.80 \\
\hline Compra de plántula de agave & & $*$ & & & & & & & 0.48 \\
\hline Flete del vivero al lugar de siembra & & $*$ & & & & & & & 0.30 \\
\hline Replante & & $*$ & & & & & & & 0.08 \\
\hline Total & & & & & & & & & 27.36 \\
\hline
\end{tabular}

Elaboración con datos de Cruz (2019).

Desde la inversión hasta la cosecha de agave existe, por lo menos, un periodo de ocho años; razón por la que la evaluación financiera del proyecto tomó esa misma ventana de tiempo. Es decir, en la investigación solo se consideró un ciclo productivo de agave. La estructura de costos en el Cuadro 2 es independiente del peso de las piñas de agave una vez terminado el ciclo productivo, por lo que un mal manejo agronómico que implique un peso bajo en el producto final tendrá emparejados costos unitarios altos. En los resultados de la investigación se ahonda en lo último. 
Respecto a los ingresos, estos se obtuvieron por hectárea y asumieron lo siguiente: a) un peso de $30 \mathrm{~kg}$ por piña en la cosecha; b) una pérdida de $10 \%$ de las plántulas de agave a lo largo de los ocho años; es decir, una pérdida de 80 plantas; y c) un precio de venta de $\$ 6.00 \mathrm{~kg}^{-1}$ de piña. Por lo que se esperó obtener un ingreso de $\$ 144000.00$ al octavo año de la puesta en marcha del proyecto en 2028.

El proyecto de producción de mezcal se consideró independiente a la producción de agave, la razón de ello fue para identificar oportunidades de negocio por separado, de tal manera que los agricultores puedan valorar que opción es más rentable y factible según su contexto. Sin embargo, en caso de querer una aproximación de la rentabilidad del proyecto en conjunto, está sería superior a la obtenida para la producción de mezcal por separado (ya que los costos serían menores al autoabastecerse de agave, el principal insumo en la estructura de costos).

El proyecto se propuso bajo un periodo de planeación de 15 años y consideró una producción anual de 3000 litros durante los primeros tres años y del doble desde el año cuatro hasta el fin del proyecto en 2035. La razón del periodo de planeación de 15 años se debió a que, en general, la región productora de agave no tiene un mercado para la reventa de activos; es decir, una vez que ha terminado la vida económica del activo esté se desecha. Por lo que, para no contemplar pérdidas innecesarias, es exactamente en un periodo de 15 años donde termina por equipararse la vida útil con la económica de la mayoría de los activos de la empresa. Respecto a la estructura de inversión y costos del proyecto de mezcal artesanal se obtuvo la información dispuesta en los Cuadros 3 a 5 , respectivamente.

En el Cuadro 3 se resalta el hecho de que el principal concepto de inversión del proyecto fue la adquisición de activos fijos con $61.01 \%$ del total de este porcentaje, la mayor parte se va a equipo auxiliar y complementario (rueda de piedra, tina de cobre y alambique) 63.26\%, seguido de los animales de carga para mover el molino de piedra con $19.17 \%$ y finalmente, la obra civil (construcción de horno, era y elaboración de registros) con $17.57 \%$.

Cuadro 3. Inversión necesaria para la producción de mezcal (miles de pesos).

\begin{tabular}{lcc}
\hline & Conceptos & Total \\
\hline 1 & Activos fijos & 156.50 \\
2 & Obra civil & 27.50 \\
3 & Animales de carga & 30.00 \\
& Equipo auxiliar y complementario & 99.00 \\
1 & subtotal & 156.50 \\
2 & Activos diferidos & 100.00 \\
& Constitución de la empresa & 10.00 \\
& Contratos por renta del terreno & 90.00 \\
\hline
\end{tabular}

En el Cuadro 4 se exponen los componentes de los costos fijos y variables, y en el Cuadro 5 se agregaron de acuerdo con el periodo de planeación. Los costos fijos significaron, para todos los años, un poco más de $15 \%$ del total (la mayor parte del presupuesto se asignó para la compra de 
leña y la manutención del animal de carga) mientras que los costos variables fueron los más importantes con poco menos de $75 \%$ del total (los principales conceptos de gasto fueron las piñas de agave y las botellas de vidrio para el empaque).

Cuadro 4. Componentes de los costos fijos y variables de la producción de mezcal.

\begin{tabular}{cccc}
\hline Descripción de costos & Concepto & Costo por concepto & Temporalidad \\
\hline & Costos variables & & \\
Materias primas: Piñas & $6750 \mathrm{~kg}$ & 6 & mensual \\
Botellas de vidrio & 250 botellas & 20 & mensual \\
Personal para acarreo de piedra y leña & 3 jornales & 150 & mensual \\
Personal para horneada & 2 jornales & 450 & mensual \\
Personal para picar y rebanar piña & 2 jornales & 150 & mensual \\
Personal en fermentación & 2 jornales & 150 & mensual \\
Personal en destilación & 2 jornales & 200 & mensual \\
Personal en refinación & 2 jornales & 200 & mensual \\
& Costos fijos & & \\
Manutención de mula & alimento & 2145 & mensual \\
Leña & 25 & 250 & mensual \\
Flete de materiales & 1 flete & 300 & mensual \\
Mantenimiento de poliducto & 1 mantenimiento & 1500 & anual \\
\hline
\end{tabular}

Cuadro 5. Estructura de costos de la producción de mezcal (miles de pesos).

\begin{tabular}{lcccccccc}
\hline \multirow{2}{*}{ Costos } & \multicolumn{3}{c}{ Capacidad inicial } & & \multicolumn{3}{c}{ Capacidad plena } \\
\cline { 2 - 4 } \cline { 7 - 9 } & $100 \%$ & $100 \%$ & $100 \%$ & & $200 \%$ & $200 \%$ & $200 \%$ \\
& 1 & 2 & 3 & & 4 & 14 & 15 \\
\hline Costos variables & 579.00 & 579.00 & 579.00 & & 158.00 & 1158.00 & 1158.00 \\
Costos fijos & 105.84 & 105.84 & 105.84 & & 184.44 & 184.44 & 184.44 \\
Costos de operación & 684.84 & 684.84 & 684.84 & & 1342.44 & 1342.44 & 1342.44 \\
\hline
\end{tabular}

Por el tamaño del cuadro se omite la información de los años cinco al trece del proyecto, los datos respectivos son idénticos a los expuestos para los años cuatro, 14 y 15.

Finalmente, en el Cuadro 6 se exponen los ingresos obtenidos para la producción de mezcal. Al igual que en el cuadro previo se omitió la presentación de los datos para los años cinco a trece.

Cuadro 6. Ingresos supuestos de la producción de mezcal.

\begin{tabular}{lcccccccc}
\hline & & \multicolumn{3}{c}{ Capacidad inicial } & & \multicolumn{3}{c}{ Capacidad plena } \\
\cline { 3 - 4 } \cline { 7 - 8 } Concepto & Unidad & $100 \%$ & $100 \%$ & $100 \%$ & & $200 \%$ & $200 \%$ & $200 \%$ \\
& & 1 & 2 & 3 & & 4 & 14 & 15 \\
\hline Botellas de mezcal 1 L & Miles de botellas & 3.00 & 3.00 & 3.00 & & 6.00 & 6.00 & 6.00 \\
Ingresos totales* & Miles de pesos & 900.00 & 900.00 & 900.00 & & 1800.00 & 1800.00 & 1800.00 \\
\hline "= se supone un precio de venta de \$300.00 por botella. Nota: por el tamaño del cuadro se omite la información de los \\
años cinco al trece del proyecto; los datos respectivos son idénticos a los expuestos para los años cuatro, 14 y 15.
\end{tabular}


Al contrastar los totales de costos e ingresos en los cuadros 5 y 6 , respectivamente, hay un diferencial positivo; es decir, año con año el proyecto genera una utilidad superior a los \$200 000.00 los primeros tres años y aun mayor al doble en los sucesivos, siendo este un buen proyecto respecto a su rentabilidad. Si bien lo anterior, proporcionó una noción ambigua de que tanto el proyecto de mezcal como el de producción de agave pudieran ser rentables, su medición precisa consistió en el cálculo de los indicadores financieros más habituales, que son (De la Torre y Zamarrón, 2002; Muñante, 2002; Baca, 2013):

Valor actual neto (VAN), $\mathrm{VAN}=\sum_{\mathrm{t}=1}^{\mathrm{T}} \frac{\mathrm{B}_{\mathrm{t}}-\mathrm{C}_{\mathrm{t}}}{(1+\mathrm{r})^{\mathrm{t}}} \mathrm{I}$; Relación beneficio-costo (RBC), $\mathrm{RBC}=\left(\sum_{\mathrm{t}=1}^{\mathrm{T}} \frac{\mathrm{B}_{\mathrm{t}}}{(1+\mathrm{r})^{\mathrm{t}}}\right) /\left(\sum_{\mathrm{t}=1}^{\mathrm{T}} \frac{\mathrm{C}_{\mathrm{t}}}{(1+\mathrm{r})^{\mathrm{t}}}\right)$; Tasa interna de retorno $(\mathrm{TIR}), \mathrm{TIR}=\mathrm{r}$ tal que VAN $=0$.

Donde: $\mathrm{B}_{\mathrm{t}} \mathrm{y}_{\mathrm{t}}$ son, respectivamente, los beneficios y costos del proyecto para el año t, I es la inversión inicial y r es la tasa de descuento del proyecto. Si bien para esté ultimo parámetro no hay un consenso sobre su valor, más bien es una decisión discrecional del evaluador, en esta investigación se propuso $\mathrm{r}=0.14$, que se consideró suficiente al incorporar la inflación, el riesgo del proyecto y un margen mínimo de ganancia.

En la evaluación del proyecto se tomó una vía netamente financiera o Costo-Beneficio (CEPAL, 2004); es decir, solo se consideraron los beneficios y costos del proyecto a precios de mercado (Duarte et al., 2007). Sin embargo, no se descartó el impacto en el desarrollo, debido a que se consideró que los proyectos, por su demanda de insumos y cobertura dentro de la región, pueden intervenir en ámbitos o dimensiones específicas de la realidad para mejorarla y con ello coadyubar al desarrollo comunitario (Rodríguez y Zeballos, 2007). En la siguiente sección del manuscrito se exponen los principales resultados de la investigación donde se incorporan los indicadores antes listados.

\section{Resultados y discusión}

En el Cuadro 7 se exponen los resultados del análisis financiero por hectárea para la producción de agave con base en la estructura de costos del Cuadro 2 y la obtención de un ingreso de $\$ 144000.00$ al octavo año de la puesta en marcha del proyecto; se consideró un peso final de piña de agave de $30 \mathrm{~kg}$ y precio de $\$ 6.00 \mathrm{~kg}^{-1}$.

Cuadro 7. Análisis financiero de la producción de agave (miles de pesos).

\begin{tabular}{lllllllll}
\hline \multirow{2}{*}{ Conceptos } & \multicolumn{1}{c}{ Año } \\
\cline { 2 - 9 } & 1 & 2 & 3 & 4 & 5 & 6 & 7 & 8 \\
\hline Ingresos totales & 0 & 0 & 0 & 0 & 0 & 0 & 0 & 144.00 \\
Costos totales & 12.10 & 3.56 & 2.70 & 2.70 & 2.70 & 2.70 & 2.70 & 0.30 \\
Flujo de efectivo & -12.10 & -3.56 & -2.70 & -2.70 & -2.70 & -2.70 & -2.70 & 143.70 \\
Ingresos totales actualizados & 0.00 & 0.00 & 0.00 & 0.00 & 0.00 & 0.00 & 0.00 & 57.55 \\
Costos totales actualizados & 12.10 & 3.12 & 2.08 & 1.82 & 1.60 & 1.40 & 1.23 & 0.12 \\
\hline
\end{tabular}




\begin{tabular}{|c|c|c|c|c|c|c|c|c|}
\hline \multirow{2}{*}{ Conceptos } & \multicolumn{8}{|c|}{ Año } \\
\hline & 1 & 2 & 3 & 4 & 5 & 6 & 7 & 8 \\
\hline Flujo de efectivo actualizado & -12.10 & -3.12 & -2.08 & -1.82 & -1.60 & -1.40 & -1.23 & 57.43 \\
\hline VAN & \multicolumn{8}{|c|}{34.07} \\
\hline $\mathrm{RBC}$ & \multicolumn{8}{|c|}{2.54} \\
\hline TIR & \multicolumn{8}{|c|}{$33 \%$} \\
\hline
\end{tabular}

De acuerdo con el Cuadro 7, la producción de agave permitiría a los agricultores que decidieran invertir en el proyecto una ganancia neta de \$34 074.09 por hectárea, además de acuerdo con su estructura de costos y la RBC, se infirió que por cada peso gastado en el proyecto se lograría recuperarlo y además se tendría un retorno de $\$ 1.54$. La TIR del proyecto indicó que su rentabilidad máxima se encuentra alrededor de $33 \%$, superior a la tasa de descuento considerada de $14 \%$.

En el Cuadro 8 se dispone el análisis de sensibilidad del proyecto, resultado de considerar distintos pesos y precios de venta en las piñas de agave. Por ejemplo, bajo un peso final de piña de $30 \mathrm{~kg}$ y una fluctuación en el precio de venta de $\$ 6.00$ (Cuadro 7) a $\$ 4.00 \mathrm{~kg}^{-1}$ (hilera 10 Cuadro 8) el VAN del proyecto caería un $56.30 \%$. Por su parte, si el precio de venta se fijase en $\$ 6.00 \mathrm{~kg}^{-1}$ y el peso de las piñas pasará de 30 a $15 \mathrm{~kg}$; es decir, se redujera $50 \%$, el VAN caería un $84.45 \%$.

Cuadro 8. Indicadores financieros de la producción de agave a distintos pesos y precios de compra.

\begin{tabular}{ccccc}
\hline Peso $(\mathrm{kg})$ & Precio $(\$)$ & VAN $(\$)$ & TIR $(\%)$ & RBC \\
\hline 30 & 7.00 & 43665.39 & 37 & 2.86 \\
25 & 7.00 & 32475.55 & 32 & 2.38 \\
15 & 7.00 & 10095.86 & 21 & 1.43 \\
25 & 6.00 & 24482.80 & 29 & 2.04 \\
20 & 6.00 & 14891.50 & 24 & 1.63 \\
15 & 6.00 & 5300.21 & 18 & 1.23 \\
25 & 5.00 & 16490.05 & 25 & 1.7 \\
20 & 5.00 & 8497.31 & 20 & 1.36 \\
15 & 5.00 & 504.56 & 14 & 1.02 \\
30 & 4.00 & 14891.50 & 24 & 1.63 \\
25 & 4.00 & 8497.31 & 20 & 1.36 \\
15 & 4.00 & -4291.09 & 10 & 0.82 \\
\hline
\end{tabular}

Cifras en rojo son indicativo de combinaciones peso-precio en las cuales la producción de agave no resulta rentable.

Con base en los hallazgos, si bien la producción de agave es rentable, su éxito se encuentra mayormente relacionado al manejo agronómico que a las condiciones de mercado, debido a que variaciones de los pesos finales de las piñas de agave se asociaron a contracciones mayores en los indicadores financieros. 
En el Cuadro 9 se disponen los beneficios y costos actualizados, e indicadores financieros de una finca productora de mezcal con una capacidad de producción anual de 3000 litros durante los primeros tres años del proyecto y del doble hasta el año 15.

Cuadro 9. Análisis financiero de la producción de mezcal a valores actualizados (miles de pesos).

\begin{tabular}{ccccccccc}
\hline Conceptos & \multicolumn{10}{c}{ Operación } \\
\hline Año & 0 & 1 & 2 & 3 & 4 & 5 & 6 & 7 \\
Costos & 256.50 & 600.74 & 526.96 & 529.07 & 909.93 & 805.98 & 700.16 & 614.18 \\
Beneficios & 0.00 & 789.47 & 692.52 & 607.47 & 1065.74 & 934.86 & 820.06 & 719.35 \\
Flujo de efectivo & -256.50 & 188.74 & 165.56 & 78.4 & 155.81 & 128.89 & 119.89 & 105.17 \\
Año & 8 & 9 & 10 & 11 & 12 & 13 & 14 & 15 \\
Costos & 538.75 & 477.2 & 414.55 & 366.96 & 318.99 & 285.09 & 245.45 & 215.31 \\
Beneficios & 631.01 & 553.51 & 485.54 & 425.91 & 373.61 & 327.72 & 287.48 & 257.68 \\
Flujo de efectivo & 92.25 & 76.31 & 70.99 & 58.96 & 54.62 & 42.63 & 42.03 & 42.38 \\
VAN & $\$ 1911.79$ & & & & & & & \\
RBC & 1.27 & & & & & & & \\
TIR & $91.98 \%$ & & & & & & & \\
\hline
\end{tabular}

Salvo por el año cero del proyecto, en los 15 años se obtuvieron flujos de efectivo positivos. Así, la inversión en el proyecto significó una ganancia neta de \$1 911 792.96. Respecto a su RBC, se tuvo que por cada peso gastado en la estructura de costos el proyecto permite recuperarlo y obtener \$0.27 adicional. Finalmente, la medida del TIR nos indicó que la rentabilidad máxima del proyecto es del $91.98 \%$.

Al igual que para la producción de agave (Cuadro 8), se implementó el análisis de sensibilidad de la rentabilidad de producción de mezcal. Para ello, se ajustó al alza el costo de las piñas de agave y el de las botellas de envasado, debido a su importancia en la estructura de los costos (Cuadro 4). En el Cuadro 10 se disponen los resultados.

Cuadro 10. Análisis financiero de sensibilidad de la producción de mezcal.

\begin{tabular}{ccc}
\hline Conceptos & $\begin{array}{c}\text { Aumento de } 20 \% \text { en precio de } \\
\text { piña }\end{array}$ & $\begin{array}{c}\text { Aumento de } 20 \% \text { en precio de } \\
\text { botellas }\end{array}$ \\
\hline VAN (miles de pesos) & $\$ 943.42$ & $\$ 1792.24$ \\
RBC & 1.12 & 1.25 \\
TIR & $54.92 \%$ & $87.46 \%$ \\
\hline
\end{tabular}

Si bien las fluctuaciones de $20 \%$ en el precio de piñas de agave y botellas de vidrio impactaron negativamente la rentabilidad del proyecto (expuesto en el Cuadro 9 el VAN se redujo un $50.65 \mathrm{y}$ $6.25 \%$, respectivamente), el proyecto no deja de ser factible financieramente, ya que en ambos casos el indicador resultó mayor a cero. Por lo tanto, aun al considerarse diversos escenarios adversos en el análisis de sensibilidad, la decisión final, tanto del proyecto de agave como de mezcal, son rentables financieramente; ya que se obtuvieron valores VAN $>0, \mathrm{RBC}>1$ y TIR $>\mathrm{r}=14 \%$. 


\section{Conclusiones}

El análisis financiero de la puesta en marcha de proyectos de agave y mezcal en Caltepec, Puebla, arrojó resultados positivos. En particular, la inversión en la producción de agave permitiría obtener una ganancia de \$34 $074.09 \mathrm{ha}^{-1}$, valor superior a lo que obtienen los agricultores por la siembra y venta de gramíneas. En Caltepec los agricultores podrían obtener $\$ 5610 \mathrm{t}^{-1}$ como máximo, lo que daría una ganancia promedio por ha de $\$ 1430.55$ anual, que a ocho años significaría una ganancia menor a $\$ 11444.40$, valor que representa cerca de $1 / 3$ de lo que se obtendría con el proyecto de producción de agave.

Sin embargo, debe tomarse en cuenta que la rentabilidad del proyecto está condicionada al peso final de las piñas de agave, por lo que es necesario invertir en tecnologías y capital humano, que permitan hacer un manejo agronómico óptimo y dé como resultado piñas de agave de buen tamaño después de ocho años de implementada la producción. Ahora bien, en lo que respecta al proyecto de mezcal, este también resulta rentable al obtener un VAN $=\$ 1$ 911,792.96. Contrastando este valor con lo que el agricultor podría obtener por la venta de maíz durante 15 años, el retorno del mezcal representaría más de 54 veces dicho valor.

Se resalta el hecho de que esta agroindustria resultaría rentable financieramente en escenarios donde el precio de sus principales materias primas: piñas de agave y botellas de envasado, incrementan $20 \%$ su coste. Sin embargo, sus indicadores podrían verse reducidos hasta $50 \%$. En suma, es posible afirmar que, bajo su correcta implementación, la producción de agave y mezcal son alternativas importantes para mejorar el ingreso de los individuos y se consideran como una gran oportunidad de desarrollo económico en las comunidades pobres y marginadas, como Caltepec, Puebla. Con respecto a la producción y comercialización del mezcal, la producción industrial genera más beneficios rentistas que la agrícola (Plascencia y Peralta, 2018).

Adicionalmente se destaca la relevancia que tienen los buenos manejos agronómicos y alianzas estratégicas para aminorar los costos en la adquisición de materias primas; sin estos las explotaciones de agave y mezcal, respectivamente, verían comprometidas sus ganancias futuras. Por lo que la puesta en marcha de los proyectos en Caltepec, Puebla, debe orientarse hacia la incorporación de tales factores.

\section{Literatura citada}

AGARED. 2017. Panorama del aprovechamiento de los Agaves en México. Guadalajara, México. Baca, G. 2013. Evaluación de proyectos. McGraw Hill. Séptima edición. México.

Cárcamo, B. y Noriega, G. 2009. Producción artesanal y tradicional de mezcal. En SIPIG-UNAM (Eds) Estado del desarrollo económico y social de los pueblos indígenas de Guerrero. México.

CEPAL. 2004. Manual: formulación, evaluación y monitoreo de proyectos sociales . División de Desarrollo Social-CEPAL. México.

CONEVAL. 2010. Medición de la pobreza: indicadores de pobreza por municipio. http://web.coneval.gob.mx/Medicion/Paginas/Medición/Anexo-estadístico-municipal2010.aspx. 
Cruz, J. 2019. Prácticas culturales en plantas de mezcal para el municipio de Caltepec, Puebla. México.

De-Torre, J. y Zamarrón, B. 2002. Evaluación de proyectos de inversión. Pearson Edu. México.

Duarte, T.; Jimenez, R. y Ruiz, M. 2007. Análisis económico de proyectos de inversión. Scientia et Technica. 333-338 pp.

Financiera Rural. 2011. Monografía del Mezcal. México.https://embamex.sre.gob.mx/ belice/images/stories/docs/mezcal.pdf.

García, J. 2010. México, país de magueyes. https://www.jornada.com.mx/2012/02/18/campais.html.

INEGI. 2020. Banco de información económica. CDMX, México. https://www.inegi.org.mx/ sistemas/bie/.

INFADED. 2019. Base de datos de Caltepec, Puebla. http://siglo.inafed.gob.mx/enciclopedia/ EMM21puebla/municipios/21027a.html.

Jiménez-Merino, A. 2017. Historia y potencial del maguey mezcalero en Puebla. http://www.econsulta.com/opinion/2017-03-12/historia-ypotencial-del-magueymezcalero-en-puebla.

Muñante, D. D. 2002. Manual de formulación y evaluación de proyectos. UACH, Mex.

Pérez, E.; Chávez, M. y González, J. 2016. Revisión del agave y el mezcal. Revista Colombiana de Biotecnología. 2:148-164.

Plascencia, M., y Peralta, L. 2018. Análisis histórico de los mezcales y su situación actual desde una perspectiva ecomarxista. Revista de Desarrollo Económico Territorial.

Rincón, C.; Navarro, E.; Herrera, J. and Bravo, A. 2015. Evaluation of the distilled quality of agave poblano produced artisanally. Revista de Ciencias Naturales y Agropecuarias. 204-212 pp.

Rodríguez, J. y Zeballos, M. 2007. Evaluación de proyectos de desarrollo local. Enfoques, métodos y procedimientos. Lima, Peru.

SAGARPA. 2017. Agave tequilero y mezcalero mexicano. México: planeación agrícola nacional: 2017-2030. México.

SIACON. 2020. Base de datos del Servicio de Información Agroalimentario y Pesquero (SIAP). México. https://www.gob.mx/siap/documentos/siacon-ng-161430.

Ulloa, M. y Medrano, P. 2019. Mezcal de Puebla: una tradición con mucha ciencia. Puebla, México. 